$$
D I A S-S T P-94-19
$$

\title{
Non-Trivial Non-Canonical W-Algebras from Kac-Moody Reductions.
}

\author{
G.A.T.F. da Costa and L. O'Raifeartaigh \\ Dublin Institute for Advanced Studies \\ 10, Burlington Road, Dublin 4
}

\begin{abstract}
By reducing a split $G_{2}$ Kac-Moody algebra by a non-maximal set of first-class constraints we produce $W$-algebras which (i) contain fields of negative conformal spin and (ii) are not trivial extensions of canonical $W$-algebras.
\end{abstract}

Introduction. In recent years it has been found that $\mathrm{W}$-algebras occur naturally in the reduction of Poisson-bracket Kac-Moody (KM) algebras by first-class constraints [1] [2], where the constraints consist of reducing a nilpotent subalgebra $\Gamma$ of the KM algebra to a single constant nilpotent generator $M_{-}$. With each such nilpotent element $M_{-}$is associated an $\operatorname{sl}(2)$ subalgebra $\mathcal{S}$ of the underlying Lie algebra, and to date almost all of the $\mathrm{W}$-algebras obtained by such reductions have been characterized by the fact that they have a basis corresponding to the highest weights of $\mathcal{S}$. Such W-algebras will be called canonical and they correspond to constraint algebras $\Gamma_{c}$ which are (i) positively graded with respect to $\mathcal{S}$ and (ii) have maximal dimension subject to the conditions that the constraints be first-class (see (2) below).

The remainder of the $\mathrm{W}$-algebras that have been obtained by KM reduction are direct sums of canonical $\mathrm{W}$-algebras and free-field algebras. For lack of a better name we shall call these $\mathrm{W}$-algebras quasi-canonical. The corresponding constraint subalgebras $\Gamma_{q}$ are subalgebras of the canonical constraint subalgebras $\Gamma_{c}$ in which some or all of the elements in the lowest grade are ommitted. Examples are the constraint algebras [3] in which all of the grade $\frac{1}{2}$ elements of $\Gamma_{c}$ are omitted.

The choices of $\Gamma_{c}$ and $\Gamma_{q}$ as constraint algebras $\Gamma$ were made in the previous reductions because they guarantee that the reduced KM algebra will be a $\mathrm{W}$-algebra i.e. will be differential polynomial and will have a basis consisting of a Virasoro and primary fields. However, although these choices are sufficient to guarantee this it is not clear that they are necessary. In ref. [4] the necessary conditions were investigated and some strong lower bounds on the dimensions of potential $\Gamma$ subalgebras were found. These lower bounds fall short of requiring that $\Gamma \supseteq \Gamma_{q}$ and thus create a margin for construction of a new kind of $\mathrm{W}$-algebra by reduction. We do not believe that the margin is very large but we wish to show in this paper that it is at any rate not empty, by constructing two such $\mathrm{W}$-algebras. The two $\mathrm{W}$-algebras are obtained by the reduction of a split $G_{2} \mathrm{KM}$ algebra with a $(3+2 \times 4+3 \times 1)$ embedding, using constraint algebras of the form $\hat{\Gamma}_{c}=\Gamma_{c} / \bar{\Gamma}_{\frac{3}{2}}$ and $\hat{\Gamma}_{q}=\Gamma_{q} / \bar{\Gamma}_{\frac{3}{2}}$, where the quotient is with respect to a 1-parameter invariant subalgebra of grade $\frac{3}{2}$. The $\mathrm{W}$-algebras obtained in this way differ from the canonical ones in that (i) they contain a primary field of strictly negative (minus one-half) conformal spin (ii) the subalgebras $W_{d}$ with the spin-content of the canonical algebra $W_{c}$ are non-trivial 
deformations of $W_{c}$ and (iii) $W_{d}$ does not decouple from its complement and in particular does not decouple from the negative spin field. The existence of a coupled field with negative conformal spin is rather unexpected and raises some questions about the unitarity of the quantized version [4].

The $G_{2}$ KM algebra and Constraints: We use the conventional root diagram for $G_{2}$ and consider the horizontal $s l(2)$-embedding i.e. the embedding with irreps of dimension $\{1,4,(3,1), 4,1\}$. We denote the generators of this $\operatorname{sl}(2)$ by $\left\{M_{-}, M_{0}, M_{+}\right\}$with Lie algebra $\left[M_{0}, M_{ \pm}\right]= \pm M_{ \pm},\left[M_{-}, M_{+}\right]=M_{0}$. For $G_{2}$ there is an orthogonal (vertical) $s l(2)$ with generators $\left\{Y_{-}, Y_{0}, Y_{+}\right\}$and the same Lie algebra. The remaining roots (which form two quadruplets with respect to the $M$ 's and four doublets with respect to the $Y$ 's) can then be labelled $E_{m}^{y}$ where $m$ and $y$ are the eigenvalues of $M_{0}$ and normalized so that $\left[M_{+}, E_{m}^{y}\right]=-E_{m+1}^{y}$ and $\left[M_{-}, E_{m}^{y}\right]=\frac{1}{2}(j+m)(j-m+1) E_{m-1}^{y}$ for $j=\frac{3}{2}$ and similarly for the $Y$ 's with $j=\frac{1}{2}$. A normalization of the roots compatible with these conventions is

$$
<M_{+}, M_{-}>=1 \quad<M_{0}, M_{0}>=-1 \quad<E_{-m}^{y}, E_{m}^{-y}>=(-1)^{m-\frac{1}{2}}
$$

with the $Y$ 's normalized in the same way as the $M$ 's.

We denote the $G_{2} \mathrm{KM}$ fields corresponding to the horizontal and vertical $s l(2)$ 's by $\left\{j_{-}(x), j_{0}(x), j_{+}(x)\right\}$ and $\{\bar{s}(x), y(x), s(x)\}$ respectively and the KM fields corresponding to the roots $E_{m}^{ \pm \frac{1}{2}}$ by $t_{m}(x)$ and $\bar{t}_{m}(x)$ respectively. Thus the KM current $j(x)$ can be written as

$j_{-}(x) M_{-}+j_{0}(x) M_{0}+j_{+}(x) M_{+}+\bar{s}(x) Y_{-}+y(x) Y_{0}+s(x) Y_{+}+t_{m}(x) E_{m}^{\frac{1}{2}}+\bar{t}_{m}(x) E_{m}^{-\frac{1}{2}}$

which we can represent diagrammatically as

$$
\begin{array}{cccc}
\multicolumn{4}{c}{s(x)} \\
t_{-\frac{3}{2}}(x) & t_{-\frac{1}{2}}(x) & t_{\frac{1}{2}}(x) & t_{\frac{3}{2}}(x) \\
j_{-}(x) & j_{0}(x) y(x) & j_{+}(x) \\
\bar{t}_{-\frac{3}{2}}(x) & \bar{t}_{-\frac{1}{2}}(x) \quad \bar{t}_{\frac{1}{2}}(x) & \bar{t}_{\frac{3}{2}}(x) \\
\bar{s}(x) &
\end{array}
$$

For the above KM-algebra the constraints mentioned in the Abstract are of the form

$$
j_{-}(x) \equiv<M_{+}, j(x)>=1 \quad<\tilde{\gamma}, j(x)>=0,
$$

where the constraint algebra $\Gamma$ is a semi-direct sum of the form $\Gamma=M_{+} \wedge \tilde{\Gamma}$ and $\tilde{\gamma}$ is any element of $\tilde{\Gamma}$. In order that the constraints (1) be first-class the constraint algebra $\Gamma$ must satisfy [5] the following conditions

$$
<\Gamma, \Gamma>=0 \quad w(\Gamma, \Gamma) \equiv<M_{-},[\Gamma, \Gamma]>=0 .
$$

The non-trivial components of the current then lie in $\Gamma^{\perp}$ which, on account of the second condition in (2) contains $\left[M_{-}, \Gamma\right]$. It is assumed that $M_{-}$is non-degenerate on $\Gamma$ so that $\left[M_{-}, \Gamma\right]$ has the same dimension as $\Gamma$, and then the natural gauge-fixing procedure (called the Drinfeld and Sokolov or DS procedure) is to set the components 
of the current which lie in $\left[M_{-}, \Gamma\right]$ equal to zero. In other words the DS gauge-fixing consists in supplementing the constraints (2) with the further linear constraints

$$
<\theta_{a}, j(x)>=0 \quad \text { where } \quad w\left(\theta_{a}, \gamma_{b}\right) \equiv<\theta_{a},\left[M_{-} \gamma_{b}\right]>=\delta_{a b} .
$$

The conformal invariance of the reduction (4) is established by noting that, although the first constraint in (4) is not compatible with the conformal group generated by the usual KM Sugawara Virasoro-operator $L_{K M}(j(x))$, it is compatible with the conformal group generated by the modified Virasoro operator

$$
\Lambda(j(x))=L_{K M}(j(x))-h^{\prime}(x) \quad \text { where } \quad h(x)=-j_{0}(x),
$$

the sign of $h(x)$ being chosen for later convenience. With respect to the modified Virasoro operator $(7)$ all of the KM fields are primary (with conformal weight $(m+1)$ ) except $h(x)$, whose conformal variation is of the form

$$
\delta_{c} h(x)=f(x) h^{\prime}(x)+f^{\prime}(x) h(x)-k f^{\prime \prime}(x),
$$

where $f(x)$ is the conformal parameter and $k$ is a constant proportional to the KM centre, which for convenience we normalize to -1 . Thus $h(x)$ transforms as the component of a spin-one connection, the inhomogeneous part of the conformal variation being $\delta_{I} h(x)=f^{\prime \prime}(x)$.

The different reductions for the chosen $\operatorname{sl}(2)$ embedding are characterized by the different choices of the constraint subalgebra $\Gamma$. In order to guarantee (5) and to obtain a differential polynomial $\mathrm{W}$-algebra it is usual to choose $\Gamma$ positive with respect to $M_{0}$ and for simplicity we shall follow this procedure. We shall also assume for simplicity that $\Gamma$ has an $s l(2)$ basis i.e. has a basis labelled by the $\operatorname{sl}(2)$ Casimir as well as $M_{0}$. There are then five possibilities, which can be grouped into three classes as follows:

$$
\begin{array}{llll}
\text { Class I } & \Gamma_{c}=\left\{M_{+}, \gamma_{\frac{3}{2}}, \gamma_{\frac{1}{2}}, \bar{\gamma}_{\frac{3}{2}}\right\} & \text { and } & \Gamma_{q}=\left\{M_{+}, \gamma_{\frac{3}{2}}, \bar{\gamma}_{\frac{3}{2}}\right\} \\
\text { Class II } & \hat{\Gamma}_{c}=\left\{M_{+}, \gamma_{\frac{3}{2}}, \gamma_{\frac{1}{2}}\right\} & \text { and } & \hat{\Gamma}_{q}=\left\{M_{+}, \gamma_{\frac{3}{2}}\right\}
\end{array}
$$

Class III $\quad \Gamma=M_{+}$.

where $\gamma_{m}=E_{m}^{\frac{1}{2}}$ for $m=\frac{1}{2}, \frac{3}{2}$ and $\bar{\gamma}_{\frac{3}{2}}=E_{\frac{3}{2}}^{-\frac{1}{2}}$. Note that the Class II subalgebras are obtained from the respective Class I subalgebras by omitting the base-element $\bar{\gamma}_{\frac{3}{2}}$

Classes I and III have already been discussed in detail in the literature. Class I contains the canonical and quasi-canonical W-algebras for this particular $\operatorname{sl}(2)$ embedding. The canonical $\mathrm{W}$-algebra $W_{c}$ is generated by $\left\{W_{1}, W_{2}, W_{\frac{5}{2}}\right\}$ where $W_{1}$ is an $s l(2)$ KM subalgebra generated by the spin-1 fields $\{\bar{s}, y, s\}, W_{2}$ is the Virasoro and $W_{\frac{5}{2}}$ is a spin $\frac{5}{2}$ doublet. The quasi-canonical subalgebra is generated by $\left\{W_{\frac{1}{2}}, W_{c}\right\}$, where $W_{\frac{1}{2}}$ is the spin $\frac{1}{2}$ doublet generated by the free-fields $\left\{t_{-\frac{1}{2}}, \bar{t}_{-\frac{1}{2}}\right\}$. The model of Class III is distinguished by the fact that it does not produce a W-algebra (for 
reasons that will be explained below). The question is: what happens for the models of the intermediate class II? For these models the first-class constraints are

$$
j_{-}(x)=1 \quad \bar{t}_{-\frac{3}{2}}(x)=\bar{t}_{-\frac{1}{2}}(x)=0 \quad \text { and } \quad j_{-}(x)=1 \quad \bar{t}_{-\frac{3}{2}}(x)=0
$$

respectively. What we shall show is that both these models produce $\mathrm{W}$-algebras. But they differ from the $\mathrm{W}$-algebras obtained previously because they each contain the field $t_{-\frac{3}{2}}(x)$, which has conformal weight minus one-half, and because the $\mathrm{W}$-algebra is not a direct sum of the canonical W-algebra and a complementary subalgebra (much less a free-field algebra). Indeed it does not even contain the canonical Walgebra. Accordingly, the models of Class II furnish examples of $W$-algebras which have negative spins and are not extensions of the canonical $W$-subalgebras.

Gauge-Fixed Fields: We first carry out the DS gauge-fixing for the models of Class II i.e. we gauge-transform to zero the part of the current that lies in $\left[M_{-}, \Gamma\right]$. As usual we begin by gauge-transforming the coefficient $h(x)$ of $M_{0}$ to zero by means of the gauge-transformation $e^{h(x) M_{+}}$. Under this gauge-transformation the fields change as follows:

$$
\begin{aligned}
& s(x) \quad y(x) \quad \bar{s}(x) \quad t_{-\frac{3}{2}}(x) \quad \bar{t}_{-\frac{3}{2}}(x) \quad \text { remain unchanged } \\
& j_{+}(x) \rightarrow w(x) \equiv j_{+}(x)+h^{\prime}(x)-\frac{h^{2}(x)}{2} \\
& t_{m}(x) \rightarrow u_{m}(x) \equiv \sum_{p \geq 0} \frac{h^{p}}{p !} t_{m-p} \quad m \neq \frac{-3}{2}
\end{aligned}
$$

and similarly for $\bar{t}_{m}(x)$. From the conformal variation of $h(x)$ given above it is easy to see that the non-primary part of the conformal variation of these fields are

$$
\delta w(x)=f^{\prime \prime \prime}(x) \quad \delta u_{m}(x)=f^{\prime \prime}(x) u_{m-1}(x),
$$

and similarly for the $\bar{u}_{m}(x)$. Thus $w(x)$ transforms like a spin-2 connection while the $u$ 's and $\bar{u}$ 's transform homogeneously but not primarily.

We then make gauge-transformations with respect to $\gamma_{\frac{1}{2}}$ and $\gamma_{\frac{3}{2}}$. In preparation for this we summarize the relevant $G_{2}$ commutation relations, namely

$$
\begin{gathered}
{\left[\gamma_{\frac{1}{2}}, M_{-}\right]=-2 E_{-\frac{1}{2}}^{\frac{1}{2}} \quad\left[\gamma_{\frac{1}{2}}, E_{-\frac{1}{2}}^{\frac{1}{2}}\right]=-\frac{1}{2} Y_{+} \quad\left[\gamma_{\frac{1}{2}}\left[\gamma_{\frac{1}{2}}, M_{-}\right]\right]=Y_{+}} \\
{\left[\gamma_{\frac{1}{2}}, Y_{0}\right]=-\frac{1}{2} \gamma_{\frac{1}{2}} \quad\left[\gamma_{\frac{1}{2}}, Y_{-}\right]=-\frac{1}{2} E_{\frac{1}{2}}^{-\frac{1}{2}}}
\end{gathered}
$$

and

$$
\left[\gamma_{\frac{3}{2}}, M_{-}\right]=-\frac{3}{2} \gamma_{\frac{1}{2}} \quad\left[\gamma_{\frac{3}{2}}, E_{-\frac{3}{2}}^{\frac{1}{2}}\right]=\frac{1}{2} Y_{+} \quad\left[\gamma_{\frac{3}{2}}, Y_{-}\right]=-\frac{1}{2} E_{\frac{3}{2}}^{-\frac{1}{2}}
$$

These and all other $G_{2}$ commutation relations can be obtained by using the normalizations of the $G_{2}$ generators given in the first paragraph and the complete antisymmetry of $<X[Y, Z]>$. 
From the above relations we see that with respect to gauge-transformations generated by $\alpha \gamma_{\frac{1}{2}}$ and $\beta \gamma_{\frac{3}{2}}$ we have

$$
u_{-\frac{1}{2}} \quad \rightarrow \quad u_{-\frac{1}{2}}-2 \alpha \quad u_{\frac{1}{2}} \quad \rightarrow \quad u_{\frac{1}{2}}-\left(\partial+\frac{y}{2}\right) \alpha-\frac{3}{2} \beta
$$

Since the DS gauge fixing in model $\hat{\Gamma}_{c}$ consists of gauging $u_{-\frac{1}{2}}$ and $u_{\frac{1}{2}}$ to zero we see that the appropriate gauge-parameters are

$$
\alpha^{c}=\frac{1}{2} u_{-\frac{1}{2}} \quad \text { and } \quad \beta^{c}=\frac{2}{3} v_{\frac{1}{2}}^{c} \quad \text { where } \quad v_{\frac{1}{2}}^{c}=u_{\frac{1}{2}}-\frac{1}{2}\left(\partial+\frac{y}{2}\right) u_{-\frac{1}{2}}
$$

Making a gauge-transformation with these parameters we find that the fields in the DS gauge for this model are

$$
\begin{aligned}
& s^{c} \\
& t_{\frac{3}{2}} \quad 0 \quad 0 \quad v_{\frac{3}{2}}^{c} \quad s^{c}=s-\frac{1}{4} u_{-\frac{1}{2}}^{2}+\frac{1}{3} t_{-\frac{3}{2}} v_{\frac{1}{2}}^{c} \\
& 1 \quad y \quad w^{c} \quad \quad \text { where } \quad \bar{v}_{\frac{1}{2}}^{c}=\bar{u}_{\frac{1}{2}}-\frac{1}{4} u_{-\frac{1}{2}} \bar{s} \\
& 0 \quad 0 \quad \bar{v}_{\frac{1}{2}}^{c} \quad \bar{v}_{\frac{3}{2}}^{c} \quad \bar{v}_{\frac{3}{2}}^{c}=\bar{u}_{\frac{3}{2}}-\frac{1}{3} \bar{s} v_{\frac{1}{2}}^{c}
\end{aligned}
$$

It is straightforward to compute $w^{c}$ and $v_{\frac{3}{2}}^{c}$ also but, except for the part that will be computed independently below their precise forms will not be needed.

For the model $\hat{\Gamma}_{q}$ the gauge-parameter $\alpha$ is identically zero and the DS gaugefixing consists only of gauging $u_{\frac{1}{2}}$ to zero. It is clear that the appropriate value of the gauge-parameter $\beta$ in this case is $\beta^{c}=\frac{2}{3} u_{\frac{1}{2}}$ and after the gauge-transformation with this value of $\beta$ we obtain the DS fields

$$
\begin{array}{cccccc} 
& \multicolumn{3}{c}{s^{q}} & & \\
t_{\frac{3}{2}} & u_{-\frac{1}{2}} & 0 & v_{\frac{3}{2}}^{q} & & s^{q}=s+\frac{1}{3} t_{-\frac{3}{2}} u_{\frac{1}{2}} \\
& & y & w^{q} & \text { where } & \\
0 & \bar{t}_{-\frac{1}{2}} & \bar{u}_{\frac{1}{2}} & \bar{v}_{\frac{3}{2}}^{q} & \bar{v}_{\frac{3}{2}}^{q}=\bar{u}_{\frac{3}{2}}-\frac{1}{3} \bar{s} u_{\frac{1}{2}} \\
& & & & &
\end{array}
$$

Again it is straightforward to compute $w^{q}$ and $v_{\frac{3}{2}}^{q}$ but their precise forms will not be needed. In each of the above models the final fields provide a complete basis for the gauge-invariant functions of the KM fields and hence the Poisson-bracket algebra of these fields induced by the original KM algebra closes. We call this algebra the reduced algebra. Since the fields are differential polynomials of the original KM fields it is obviously a differential polynomial algebra. Thus it will be a W-algebra if it has a basis consisting of a Virasoro operator and a set of fields which are primary with respect to it. For short we shall call such a basis a primary basis. 
Existence of a Primary Basis: We wish to show that for the two models of Class II the reduced algebra has a primary basis. First we note that the modified Virasoro operator $\Lambda(x)$ of (4) is a candidate for the Virasoro base-element since it is a differential polynomial in the $\mathrm{KM}$ current components and transforms in the correct manner. Furthermore, since $\Lambda(x)$ and $W(x)$ are the only gauge-fixed fields which are linear in $j_{+}(x)$ either one can be chosen as a base element of the reduced algebra (which implies, of course, that $\Lambda(x)$ and $W(x)$ differ only by a differential polynomial in the other gauge-fixed fields and it is easy to verify that such is indeed the case). Because of its transformation properties we choose $\Lambda(x)$.

To obtain some orientation for considering the primariness of the other base elements in the models of Class II we first consider the single model of Class III, which contains all the $u$-fields. The $u$ 's are the only fields which are not primary and even for them the fields $u_{-\frac{1}{2}}, u_{\frac{3}{2}}$ and the corresponding barred fields can be converted to primary fields by the addition of differential polynomials in fields of lower grades, namely

$$
\begin{gathered}
u_{-\frac{1}{2}} \rightarrow p_{-\frac{1}{2}} \equiv u_{-\frac{1}{2}}+2 \partial t_{-\frac{3}{2}} \\
u_{\frac{3}{2}} \rightarrow p_{\frac{3}{2}} \equiv u_{\frac{3}{2}}-\frac{2}{3} \partial u_{\frac{1}{2}}-\frac{1}{6}\left(2 \partial^{2}+3 \Lambda\right) u_{-\frac{1}{2}}+\frac{1}{9}\left(2 \partial^{3}+7 \Lambda \partial+4(\partial \Lambda)\right) t_{-\frac{3}{2}},
\end{gathered}
$$

and similarly for the barred fields. The non-primariness of the model comes from the $u_{\frac{1}{2}}$ field and its barred counterpart. The point is that, so long as $t_{-\frac{3}{2}}$ is not zero, $u_{\frac{1}{2}}$ can not be converted to a primary field. To see this we note from (12) that the $u$-fields and $w$ transform linearly with respect to the conformal group and $w$ transforms linearly with respect to the Mobius subgroup $\left(f^{\prime \prime \prime}(x)=0\right)$. Hence $u_{\frac{1}{2}}$ must be converted to a primary field even at the linear level. This can be done only by the addition of terms that are linear in the $u$ 's and $w$ and since the only fields with the correct quantum numbers are $u_{-\frac{1}{2}}^{\prime}$ and $t_{-\frac{3}{2}}^{\prime \prime}$, of which $t_{-\frac{3}{2}}^{\prime \prime}$ is Mobius-primary, the only possibility is to let $u_{\frac{1}{2}} \rightarrow U_{\frac{1}{2}}=u_{\frac{1}{2}}+\kappa u_{-\frac{1}{2}}^{\prime}$ where $\kappa$ is a constant. But from (12) it follows that the non-conformal part of the variation of $U_{\frac{1}{2}}$ with respect to the Mobius subgroup is $\delta U_{\frac{1}{2}}=\left(\left(1+\frac{\kappa}{2}\right) u_{-\frac{1}{2}}+\kappa t_{-\frac{3}{2}}^{\prime}\right) f^{\prime \prime}$, which shows that no choice of $\kappa$ can make $U_{\frac{1}{2}}$ primary and thus convert $u_{\frac{1}{2}}$ to a primary field. Similarly, unless $\bar{t}_{-\frac{3}{2}}$ is zero, the field $\bar{u}_{\frac{1}{2}}$ cannot be converted to a Mobius-primary field, much less a totally primary one. This is why the reduced algebra of model III, for which $u_{\frac{1}{2}}$ and $\bar{u}_{\frac{1}{2}}$ are base elements, is not a $W$-algebra. (The argument that primariness must hold at the linear level alone is given in more detail in [3] and the above result is actually a special case of the result that $u_{n}$ cannot be made even Mobius-primary unless $\left.u_{-(n+1)}=0\right)$.

In both models of Class II the field $\bar{t}_{-\frac{3}{2}}$ is zero so there is no problem in converting the field $\bar{u}_{\frac{1}{2}}$ to a primary field, namely by letting

$$
\bar{u}_{\frac{1}{2}} \rightarrow \bar{p}_{\frac{1}{2}} \equiv u_{\frac{1}{2}}-2 \partial \bar{t}_{-\frac{1}{2}} .
$$

The field $u_{\frac{1}{2}}$ remains non-primary but it is no longer a base-element of the reduced algebra because it is gauged away by the DS gauge-fixing corrresponding to $\gamma_{\frac{3}{2}}$. Of 
course, it reappears in other elements of the reduced algebra, but, as can be seen by inspection of (15) through (18) it appears in these elements only in the combinations

$$
\left.\left.u_{\frac{3}{2}}-\frac{2}{3} u_{\frac{1}{2}}^{\prime} \quad\left(\text { in } v_{\frac{3}{2}}\right) \quad u_{\frac{1}{2}} t_{-\frac{3}{2}} \quad \text { (in } s\right) \quad u_{\frac{1}{2}} y \quad \text { (in } v_{\frac{3}{2}}\right) \quad u_{\frac{1}{2}} \bar{s} \quad \text { (in } \bar{v}_{\frac{3}{2}} \text { ) }
$$

We now wish to show that, because $u_{\frac{1}{2}}$ appears only in these combinations, the DS basis can be replaced by a basis of primary fields. For the first expression in (22) the conversion is already given by (20). For the second expression the conversion is easily verified to be

$$
u_{\frac{1}{2}} t_{-\frac{3}{2}} \rightarrow p_{0} \equiv u_{\frac{1}{2}} t_{-\frac{3}{2}}+2 u_{-\frac{1}{2}} \partial t_{-\frac{3}{2}}+2\left(\partial t_{-\frac{3}{2}}\right)^{2} .
$$

The last two expressions in (22) can be handled together by using the general observation that if $T_{m}$ is any primary field of grade $m$ ( $\left.\operatorname{spin} m+1\right)$, such that $m \neq-1$ or $-\frac{3}{2}$ then the modified field $P_{m}$ given by

$$
P_{m} \equiv u_{\frac{1}{2}} T_{m}-\frac{1}{m+1} u_{-\frac{1}{2}} \partial T_{m}+\frac{1}{(m+1)(2 m+3)} t_{-\frac{3}{2}}\left(\partial^{2}-(m+1) \Lambda\right) T_{m},
$$

is primary. The formula for $m=0$ obviously applies to the last two expressions in (21) and thus converts these two expressions into primary fields. Since in all the above conversions only gauge-invariant fields were used and the highest spin field in each case remained unchanged it is clear that the resulting primary fields (together with the modified Virasoro) form a basis. We have thus established by construction that for both models of Class II there is a primary basis. The construction is rather ad hoc and it would be interesting to find a more systematic way of obtaining the primary basis.

General Structure of the W-Algebras. Having established that there exists a primary basis for the DS gauge-fixed fields we now wish to investigate the Poissonbracket (PB) algebra of these fields. We first note that the DS fields are gaugeinvariant polynomials of the original KM fields which reduce to independent $\mathrm{KM}$ fields in the linear limit. This means that they form a basis for all the gaugeinvariant differential polynomials of the KM fields and that the reduced algebra is a freely-generated differential polynomial algebra. By its PB-structure we shall mean the PB structure of the differential polynomials inherited from the KM PB structure

$$
\{<A, j(x)>,<B, j(y)>\}=<[A, B], j(x)>\delta(x-y)+c<A, B>\delta^{\prime}(x-y)
$$

The spin structures of the DS basis for the models $\hat{\Gamma}_{c}$ and $\hat{\Gamma}_{q}$ are of the form

$$
W=W_{d}+\left(W_{-\frac{1}{2}}+W_{\frac{3}{2}}\right) \quad \text { and } \quad W=W_{d}+W_{\frac{1}{2}}+\left(W_{-\frac{1}{2}}+W_{\frac{3}{2}}\right)
$$

respectively, where $W_{d}$ is a 6-dimensional subalgebra with the spin content of the canonical W-algebra and $W_{\frac{1}{2}}$ is a 2-dimensional subalgebra with the spin content of the extra fields that appear in the quasi-canonical model. $W_{-\frac{1}{2}}$ and $W_{\frac{3}{2}}$ are 1-dimensional and contain the two extra fields that appear in the present models. As we shall see later in (37) the pair of fields $\left\{u_{-\frac{1}{2}}, \bar{t}_{-\frac{1}{2}}\right\}$ in $W_{\frac{1}{2}}$ and the pair of fields $\left\{t_{-\frac{3}{2}}, \bar{v}_{\frac{1}{2}}\right\}$ which appear in the present models are each a conjugate pair with 
respect to the PB's. Thus the $W$-algebras consist of $W_{d}$ plus one and two conjugate pairs respectively. It would be straightforward to compute the $\mathrm{W}$-algebra in the DS basis but since the DS basis does not exhibit the direct sum structure in the quasicanonical case it probably does not exhibit the true structure in the present case. On the other hand, the large freedom in choosing a general basis (as exemplified by (28) and (29) below) makes it difficult to extract any detailed information. Hence we restrict ourselves to obtaining two key structural results, namely that $W_{d}$ is a non-trivial deformation of a canonical subalgebra and that it does not decouple from its complement.

In order to establish these results we note that since they are negative it is sufficient to establish them for any subset of field configurations. Hence, for simplicity, we restrict ourselves to the constant configurations. This has the advantage that all computations become Lie-algebraic, modulo overall delta-functions which we suppress from now on.

We also recall from [4] that in the case of the quasi-canonical subalgebra the free field algebra $W_{\frac{1}{2}}$ does not decouple from $W_{c}$ in the original DS basis but only in a modified basis. For example, the modification required for $W_{\frac{1}{2}}$ to decouple from the $W_{1}$ part of the quasi-canonical model is to change the basis of $W_{1}$ from $\{\bar{s}, y, s\}$ to $\{\bar{s}, y, s\}-\frac{1}{4}\left\{\bar{t}_{-\frac{1}{2}}^{2}, 2 \bar{t}_{-\frac{1}{2}} t_{-\frac{1}{2}}, t_{-\frac{1}{2}}^{2}\right\}$ This warns us that to establish any negative result such as no-decoupling, it is not sufficient to establish it in the DS basis, but in any permissible basis. In this connection it should be observed that there is a new phenomenon that occurs because of the negative spin field, namely that there exist scalar combinations of the DS fields. This means that coefficients in any modification of the basis may be polynomials in these scalars rather than constants. The scalars in question are $\nu=\bar{s} t_{-\frac{3}{2}}^{2}$ for both models and $\mu=t_{-\frac{3}{2}} \bar{t}_{-\frac{1}{2}}$ for model $\hat{\Gamma}_{q}$.

Deformation Structure of $W_{d}$. We first consider the subalgebras $W_{d}$ which have the spin content of the canonical W-algebra. From the results in [4] there exists a basis in which these algebra become canonical for $t_{-\frac{3}{2}}=0$ and $\bar{v}_{\frac{1}{2}}=0$. So, at worst, they are deformations of $W_{c}$. The only question is whether the deformation is trivial. Fortunately there is a property of the present $G_{2}$ embedding that permits us to determine this, namely that the spin 1 part of the canonical W-algebra must be an $\operatorname{sl}(2) \mathrm{KM}$ algebra. This means that a necessary condition for $W_{d}$ to be canonical is that $W_{1}$ be an $s l(2) \mathrm{KM}$ algebra. Hence to establish the contrary, it is sufficient to establish that there is no basis in which $W_{1}$ (which is much more tractable than $\left.W_{d}\right)$ forms an $\operatorname{sl}(2)$ subalgebra.

We first note that since the $\gamma_{\frac{3}{2}}$ gauge-fixing determines the coefficient of $t_{-\frac{3}{2}} v_{\frac{1}{2}}$ in $s^{c}$ and $s^{q}$, we have

$$
\left\{\bar{s}, s^{c}\right\}=\left\{\bar{s}, s^{q}\right\}=y+\frac{1}{3} t_{-\frac{3}{2}}^{2} \bar{v}_{\frac{1}{2}}
$$

which shows that there is no $\operatorname{sl}(2)$ structure in the DS basis. Thus we must consider the most general basis for the spin 1 sector which is

$$
\{\bar{S}, Y, S\} \quad \text { where } \quad \bar{S}=\bar{s}+a \bar{\tau}^{2} \quad Y=y+A t \bar{v}_{\frac{1}{2}}+(B \bar{\tau}+C \bar{s} t) u_{-\frac{1}{2}}
$$


and

$$
\begin{aligned}
S=\left(s+\frac{t}{3} v_{\frac{1}{2}}\right)+D u_{-\frac{1}{2}}^{2}+t E y u_{-\frac{1}{2}} & +t^{2}\left(F \Lambda+G y^{2}+H u_{-\frac{1}{2}} \bar{v}_{\frac{1}{2}}\right) \\
& +t^{3}\left(I \bar{v}_{\frac{3}{2}}+K y \bar{v}_{\frac{1}{2}}\right)+t^{4} N \bar{v}_{\frac{1}{2}}^{2}
\end{aligned} .
$$

where $t$ and $\bar{\tau}$ are abbreviations for $t_{-\frac{3}{2}}$ and $\bar{t}_{-\frac{1}{2}}$ respectively, and the coefficients $a, A, B$ etc. may be polynomials in the scalars $\mu$ and $\nu$. The expressions $v_{\frac{1}{2}}, \bar{v}_{\frac{1}{2}}$ denote $v_{\frac{1}{2}}^{c}, \bar{v}_{\frac{1}{2}}^{c}$ and $u_{\frac{1}{2}}, \bar{u}_{\frac{1}{2}}$ for the models $\hat{\Gamma}_{c}$ and $\hat{\Gamma}_{q}$ respectively, and for the model $\hat{\Gamma}_{c}$ we have $D=-\frac{1}{4}$ and $\mu=B=C=E=H=0$.

It is important to note that in constructing (28) (29) we have restricted ourselves to polynomials which form a basis for the gauge-invariant polynomials. i.e. to polynomials that remain unchanged in the linear limit. Otherwise we would be considering not the freely-generated polynomial W-algebra under consideration but the algebras generated by various classes of functions (general polynomials, rational functions etc.) of the DS base-elements, which in general have structures quite different from the W-algebra.

The condition that the spin sector should form an $s l(2) \mathrm{KM}$ algebra is that

$$
\{\bar{S}, Y\}=\bar{S} \quad\{\bar{S}, S\}=Y \quad\{Y, S\}=S .
$$

but since $\bar{S}$ is quadratic in $\tau$ and we shall finally be be restricting ourselves to configurations for which $\tau=0$, we can simplify this condition by letting $\bar{S} \rightarrow \bar{s}$. The $s l(2)$-condition then reduces to

$$
\{\bar{s}, Y\}=\bar{s} \quad\{\bar{s}, S\}=Y \quad\{Y, S\}=S .
$$

To investigate (31) we first compute some relevant KM and W Poisson-brackets, namely

$$
\left\{\bar{s}, t_{m}\right\}=\bar{t}_{m} \quad \rightarrow \quad\left\{\bar{s}, u_{-\frac{1}{2}}\right\}=\bar{\tau} \quad\left\{\bar{s}, v_{\frac{1}{2}}\right\}=\bar{v}_{\frac{1}{2}} \quad\left\{\bar{s}, \bar{v}_{\frac{3}{2}}\right\}=-\frac{1}{3} \bar{s} v_{\frac{1}{2}}
$$

For the general basis the first condition in (31) is satisfied automatically for the $\hat{\Gamma}_{c}$ model and requires that $B=C=0$ for the model $\hat{\Gamma}_{q}$. Since $\{\bar{s}, S\}$ must take the same general form as $Y$ we see that the second condition simply gives linear relations between the coefficients of $Y$ and $S$. The only relation we shall need is the relation between the coefficients of $t \bar{v}_{\frac{1}{2}}$ which is easily seen to be

$$
A=\frac{1}{3}+Q \quad \text { where } \quad Q=H \mu+\left(K-\frac{I}{3}\right) \nu-\frac{1}{2} N \mu \nu .
$$

The question then centres on the third condition in (31). Since $y$ alone reproduces $S$ the question is whether $\{\Delta y, S\}=0$ where $\Delta y \equiv Y-y$. We shall show that this Poisson-bracket is not zero by showing that it projects onto the Virasoro field (or an equivalent gauge-invariant base-element). For this it is sufficient to show that it projects onto $j_{+}$since such a base element is the only one in which $j_{+}$can occur linearly. As the only brackets that can produce $j_{+}$are

$$
\left\{t_{-\frac{1}{2}}, \bar{t}_{\frac{3}{2}}\right\} \quad\left\{\bar{t}_{\frac{1}{2}}, t_{\frac{1}{2}}\right\} \quad\left\{\bar{t}_{-\frac{1}{2}}, t_{\frac{3}{2}}\right\}
$$


and $\Delta y$ contains only $t_{-\frac{1}{2}}, \bar{t}_{-\frac{1}{2}}$ and $\bar{t}_{\frac{1}{2}}$ while $S$ does not contain $t_{\frac{3}{2}}$ we see that the coefficient of $j_{+}$in $\{\Delta y, S\}$ is

$$
\frac{\partial \Delta y}{\partial \bar{t}_{\frac{1}{2}}} \frac{\partial S}{\partial t_{\frac{1}{2}}}+\frac{\partial \Delta y}{\partial t_{-\frac{1}{2}}} \frac{\partial S}{\partial \bar{t}_{\frac{3}{2}}}=\frac{1}{3}(1-I \nu) A t^{2}+\left(B \mu+C \nu-\frac{\epsilon}{4} A \nu\right) I t^{2}
$$

where $\epsilon=1,0$ for models $\hat{\Gamma}_{c}$ and $\hat{\Gamma}_{q}$ respectively. Since this expression does not vanish even for the configurations in which $\bar{s}=\bar{\tau}=0$ (for which $\mu=\nu=0$ and hence $A=\frac{1}{3}$ from (33)) we see that $\{\Delta y, S\}$ cannot be zero. Thus there is no basis in which the spin 1 fields form an $\operatorname{sl}(2) \mathrm{KM}$ subalgebra and thus no basis in which $W_{d}$ is canonical.

With hindsight, knowing that the projection of the $W_{1}$-algebra onto the Virasoro occurs even for the configurations $\bar{s}=\bar{\tau}=u_{-\frac{1}{2}}=0$ and to order $t^{2}$ one can obtain the result in a faster and more intuitive way. If we restrict to these configurations we can omit in (28) and (29) all the terms that are quadratic in these variables. If we restrict further to terms of order $t^{2}$ and recall that for constant fields the Virasoro term can be neglected, we see that (28) (29) then reduce to

$$
\bar{S}=\bar{s} \quad Y=y+A t \bar{v}_{\frac{1}{2}} \quad S=s+\frac{t}{3} v_{\frac{1}{2}}
$$

which generalizes the DS basis only to the extent that it has the free parameter $A$. The fact there is no $\operatorname{sl}(2)$ structure then follows from the observation that there is no value of $A$ that satisfies both the second and third conditions in (30).

No Decoupling Although $W_{d}$ is only a deformation of the canonical W-algebra it might still be possible for $W_{d}$ to decouple from its complement and some further insight into the structure of the $\mathrm{W}$-algebras of these models is obtained by showing that this is not the case. A preliminary hint that $t$ does not decouple from $W_{d}$ is that the Virasoro contains a term $t_{-\frac{3}{2}} \bar{t}_{\frac{3}{2}}$ and thus there does not seem to be any way in which the Virasoro could be split into a part which acts only on $t$ and a part which acts only on $\bar{v}_{\frac{3}{2}}$.

We first recall that the complement of $W_{d}$ consists of the pairs of fields $\left\{t, \bar{v}_{\frac{1}{2}}\right\}$ and $\left\{u_{-\frac{1}{2}}, \bar{\tau}\right\}$ in the model $\Gamma_{q}$ and the first of these pairs in the model $\Gamma_{c}$. These pairs of fields are conjugate in the sense that

$$
\left\{t, \bar{v}_{\frac{1}{2}}^{c}\right\}=\frac{3}{2}\left(1+\frac{\nu}{4}\right) \quad\left\{t, \bar{u}_{\frac{1}{2}}\right\}=\frac{3}{2}(1-\mu) \quad \text { and } \quad\left\{\bar{\tau}, u_{-\frac{1}{2}}\right\}=2\left(1-\frac{\mu}{4}\right)
$$

where the first two results refer to the models models $\hat{\Gamma}_{c}$ and $\hat{\Gamma}_{q}$ respectively and the third is relevant only for the model $\hat{\Gamma}_{q}$. The fields in each conjugate pair are coupled by definition and in fact they are intrinsically coupled in the sense that no polynomial modification can make them decouple. This is because any modification would add higher-degree terms and the $\mathrm{PB}$ of $t$ with any such higher-degree terms would be at least linear in the fields and thus could not compensate the constant terms in (37). A new feature of the present models is that we then have a negative grade field, namely $t$, intrinsically coupled to a positive grade field. (This situation actually generalizes to any DS model with negative spin fields $W_{-s}$ because if such 
fields survive the constraint and gauge-fixing conditions so do their $M_{-}$conjugate fields $W_{s+1}$, and the PB of the two conjugate elements contains a non-zero constant).

Since the conjugate pairs are intrinsically coupled the question then is whether $W_{d}$ can decouple from the conjugate pairs. Let us first consider the pair $\left\{t, \bar{v}_{\frac{1}{2}}\right\}$ which occurs in both models. Using (37) and $\left\{t, u_{m}\right\}=-\frac{3}{2} t u_{m-1}$ for $m \leq \frac{1}{2}$ one finds that

$$
\{t, Y\}=\left(\left(A\left(1+\frac{\nu}{4}\right)-\frac{1}{3}\right) \frac{3 t}{2} \quad \text { and } \quad\{t, Y\}=\left(\left(A-\frac{1}{3}\right)-(A+B) \mu-C \nu\right) \frac{3 t}{2}\right.
$$

for the models $\hat{\Gamma}_{c}$ and $\hat{\Gamma}_{q}$ respectively. From (38) we see that in the first model there is no polynomial value of the parameters for which $t$ decouples.

In the second model $t$ decouples from $Y$ for $A=-B=\frac{1}{3}$ and $C=0$ i.e. for $Y=$ $y+\frac{t}{3} \bar{u}_{\frac{1}{2}}-\frac{\bar{\tau}}{3} u_{-\frac{1}{2}}$ and one can check that it also decouples from $S=s+\frac{t}{3} u_{\frac{1}{2}}-\frac{1}{6} u_{-\frac{1}{2}}^{2}$. So there is a choice of parameters for which $t$ does not couple directly to the spin 1 sector. However, for the conjugate field, which reduces to $\bar{u}_{\frac{1}{2}}$ in this model, we have

$$
\left\{Y, \bar{u}_{\frac{1}{2}}\right\}=\left\{y+\frac{t}{3} \bar{u}_{\frac{1}{2}}-\frac{\bar{\tau}}{3} u_{-\frac{1}{2}}, \bar{u}_{\frac{1}{2}}\right\}=-\frac{\mu}{2} \bar{u}_{\frac{1}{2}}+\ldots
$$

where the dots denote terms not containing $\bar{u}_{\frac{1}{2}}$, which shows that $\bar{u}_{\frac{1}{2}}$ couples to $Y$. Furthermore, this coupling cannot be compensated by a polynomial modification of $\bar{u}_{\frac{1}{2}}$ because the most general polynomial modification is to add terms of the form $y \frac{\overline{2}}{\bar{\tau}}, \bar{s} u_{-\frac{1}{2}}$ and $\bar{s} y t$ and the part of the PB's of these terms with $Y$ which contain $\bar{u}_{\frac{1}{2}}$ vanish on the surface $\bar{s}=y=0$ where the coefficient of $\bar{u}_{\frac{1}{2}}$ in (39) does not vanish. Thus in the model $\hat{\Gamma}_{q}$ the field $\bar{u}_{\frac{1}{2}}$ couples directly to the spin 1 sector and the field $t$ then couples indirectly.

Let us next consider the conjugate pair $\left\{\bar{\tau}, u_{-\frac{1}{2}},\right\}$, which occurs only for the model $\hat{\Gamma}_{q}$. This pair is of interest because it decouples in the quasi-canonical model $\Gamma_{q}$. To see whether it decouples in the model $\hat{\Gamma}_{q}$ we compute the PB of $\bar{s}+a \bar{\tau}^{2}$ and $u_{-\frac{1}{2}}+b y t$, which are the most general fields that can be constructed corresponding to the base elements $\bar{s}$ and $u_{-\frac{1}{2}}$. We obtain

$$
\left\{\bar{s}+a \bar{\tau}^{2}, u_{-\frac{1}{2}}+b y t\right\}=[(1+4 a) \overline{+} a(b-1) \mu] \bar{\tau}+b \bar{s} t .
$$

In the quasi-canonical case for which $t=0$ this vanishes for $4 a=-1$ but in the present case there is clearly no polynomial choice of parameters for which it vanishes. Thus there is no basis in which this pair decouples from $W_{d}$.

From the above results we conclude that for neither of the present models is there a basis in which $W_{d}$ decouples from its complement.

Summary and Comments: We have constructed two W-algebras by the reduction of a $G_{2}$ Kac-Moody algebra, using a non-principal $s l(2)$ embedding and constraint algebras which are $s l(2)$ positive and are proper subalgebras of the canonical constraint algebra. Both W-algebras have a field of strictly negative conformal weight and a subalgebra $W_{d}$ which is a deformation of the canonical $\mathrm{W}$-algebra. The subalgebra $W_{d}$ does not decouple from its complement or even from the negative spin 
field. Indeed the $\mathrm{W}$-algebra would appear to be indecomposable. We have not given the details of the W-algebra, partly because this could be done easily only in the DS basis which is not primary and is not expected to exhibit the structure in its simplest form and partly because the details are not of any great interest at present. The primary basis has to be constructed from the DS basis in an ad hoc manner and an interesting question is to whether one could find a systematic procedure for obtaining the primary basis directly for this kind of model. The appearance of negative spin fields which do not decouple is a little surprising as it would seem to imply that the quantized version of the theory could not be unitary (the Virasoro operator could not be self-adjoint [5]) but it remains to be seen whether this is really the case. Finally it should be mentioned that the results would be essentially the same even if the Virasoro operator were not unique since the reduced algebras would still be Walgebras with respect to $\Lambda$ and the non-decoupling and $\operatorname{sl}(2)$ non-closure results are statements about base elements which do not depend strongly on the spin-labelling.

Acknowledgement The authors wish to thank Dr. I. Tsutsui and the referee for some valuable criticisms and suggestions.

References

[1] L. Feher, L. O'Raifeartaigh, P. Ruelle, I. Tsutsui and A. Wipf, Physics Reports 222(1992)1-64 and references therein.

[2] P. Bouwknecht and K. Schoutens, Physics Reports 223(1993)183 and references therein.

[3] L. Feher, L. O'Raifeartaigh, P. Ruelle and I. Tsutsui, Comm. Math. Phys. (in press)

[4] J. de Boer and T. Tjin, Comm. Math. Phys. 160(1994)317 P. Bowcock and G. Watts, Nucl. Phys. 379B(1992) 63-96

[5] P. Goddard and D. Olive, Int. J. Mod. Phys. A1 (1986) 303. 